\section{Extra genes, in and out of chromosomes}

\section{Joseph G. Gall}

Gene Amplification. Edited by Robert T. Schimke. Pp.339. ISBN 0-87969-151-4. (Cold Spring Harbor Laboratory: 1982.) \$35 (US), \$42 (elsewhere).

WHEN mammalian tissue cultures are treated with gradually increasing concentrations of methotrexate (MTX), cell lines can be selected that are resistant to very high concentrations of the otherwise toxic drug. The basis of resistance may be decreased permeability of the cells to the drug or decreased binding between the drug and the target enzyme, dihydrofolate reductase (DHFR). In a significant number of lines, however, resistance is due to compensatory production of the enzyme, which itself is related to increased synthesis of the mRNA and ultimately to differential replication or amplification of the gene coding for DHFR.

These surprising facts were first reported four years ago in elegant experiments by Robert Schimke and his colleagues at Stanford University. Progress in the field has been rapid since then, and late last year Schimke organized a small symposium at Cold Spring Harbor to summarize that progress. This volume, Gene Amplification, contains 42 short papers based on presentations at the symposium.

Many of the papers are concerned with the chromosomal and molecular state of the amplified genes. It became evident early on that two unusual cytological phenomena could be associated with amplified cell lines: first, the presence of small acentric chromosomes, which, because they often occur in pairs, are called double minutes; and second the appearance of long chromosome segments with little evidence of G-banding, referred to as homogeneously staining regions. Amplified genes have now been demonstrated in both regions by in situ nucleic acid hybridization. There is a good but not perfect correlation between stability of the amplified condition (when MTX is removed) and the physical location of the amplified genes - homogeneously staining regions being characteristic of stable lines, double minutes of unstable ones. It is possible, therefore, that amplified gene copies appear first as small extrachromosomal elements that later become stably integrated into one or more chromosomes. The size of the amplified region has been measured by hybridizing DNA from amplified lines with genomic sequences that flank the DHFR gene. The amplified segment may be up to $100 \mathrm{~kb}$ in length, considerably longer than the coding region itself ( $31 \mathrm{~kb}$ counting its five introns).

Almost nothing is known about the initial molecular events of amplification in tissue cultures. A model discussed in the book by Botchan and by Spradling involves multiple rounds of replication at a single origin of replication, giving rise to an "onion-skin" configuration. An attractive feature of this model is the ease with which it can be applied to the chorion genes of Drosophila, whose amplification in the follicle cells of the oocyte has been analysed in detail by Spradling. In this case the chorion genes are more highly amplified than surrounding sequences, the level of amplification falling off progressively on both sides of the genes. This is just what one would expect if multiple replication forks move away from an origin of replication in or near the genes.

Several other specific amplifications have been induced in tissue cultures, of which the best characterized involve resistance to $N$-(phosphonacetyl)-Laspartate or to the cadmium ion. These cases involve overproduction of the multifunctional protein "CAD" and of metallothionein-1 respectively. Several other likely cases are discussed in the book, and it seems probable that many genes can be amplified if a suitable inhibitor of enzyme activity is available. An interesting possibility is that random genomic fragments regularly detach from chromosomes and require only a selective force to undergo differential replication.

All 42 articles in the volume are short; indeed some are little more than expanded abstracts. The quality is variable, and since many are rather specialized the general reader will have to pick and choose to avoid getting bogged down in cytological and molecular detail. In addition, the title Gene Amplification is somewhat misleading. Schimke's major interest is drug-induced gene amplification in cultured cells, and that is what the book really covers. Gene amplification as a general biological phenomenon is touched upon, but readers unfamiliar with the field would not guess that naturally occurring gene amplification, particularly of rDNA, has been investigated extensively during the past 15 years in a variety of vertebrates, insects and protozoa. For a broader introduction to the topic one should begin with Adrian Bird's review article, "Gene reiteration and gene amplification", published in Vol.III of Prescott and Goldstein's Cell Biology (Academic, 1980).

Nevertheless this volume summarizes a large amount of work on an important topic. Like the much larger 1980 Cold Spring Harbor Symposium on movable genetic elements, it emphasizes the plasticity of the eukaryotic genome, a subject of great current interest. Because the book was published just six months after the meeting itself, the articles are timely and will prove useful to a wide audience.

Joseph G. Gall is Professor of Biology, Molecular Biophysics and Biochemistry at Yale University.

\section{Centrifugal Microfilters}

Bioanalytical Systems introduces a centrifugal microfilter for membrane filtration of small volumes using the force of a conventional bench top centrifuge. Ideal for LC sample preparation, extraction of TLC spots, and many other applications.

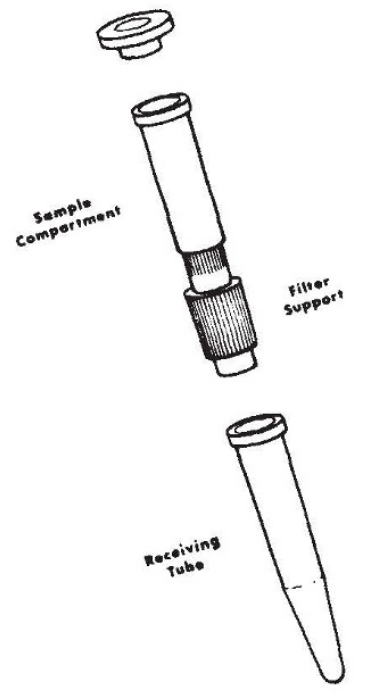

- minimize loss and contamination

- no vacuum or pressure required

- solvent resistant polyalomar

- filter many samples simultaneously

- a variety of filter materials are available

- reusable

Send for details...

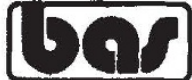

BIOANALYTICAL SYSTEMS INC. 